\title{
Management of \\ unruptured intracranial aneurysms
}

\author{
Lindsy N. Williams, MD \\ Robert D. Brown, Jr., MD, MPH
}

\section{Summary}

Unruptured intracranial aneurysms (UIA) are a common finding, occurring in about $2 \%$ of the population, making them very likely to be seen by most practitioners, and present a challenge in the recommendations for optimal management and screening. The consequences of aneurysm rupture are dire, with high likelihood of significant morbidity and mortality. Most aneurysms do not rupture and patients harboring these lesions often remain asymptomatic. There are effective surgical and endovascular interventions to prevent rupture, but these procedures carry a risk of adverse complications. This article addresses the challenges of screening and management of UIAs.

I

ntracranial saccular or berry aneurysms are acquired vascular lesions consisting of thinned outpouchings of the arterial wall. They are most commonly seen at the bifurcation of arteries in the proximal circle of Willis (figure 1). The estimated prevalence of unruptured intracranial aneurysms is $2 \%-3 \%$ in the general population, but may be higher in older patients, females, and patients with family history or certain genetic conditions. ${ }^{1}$ Aneurysms can

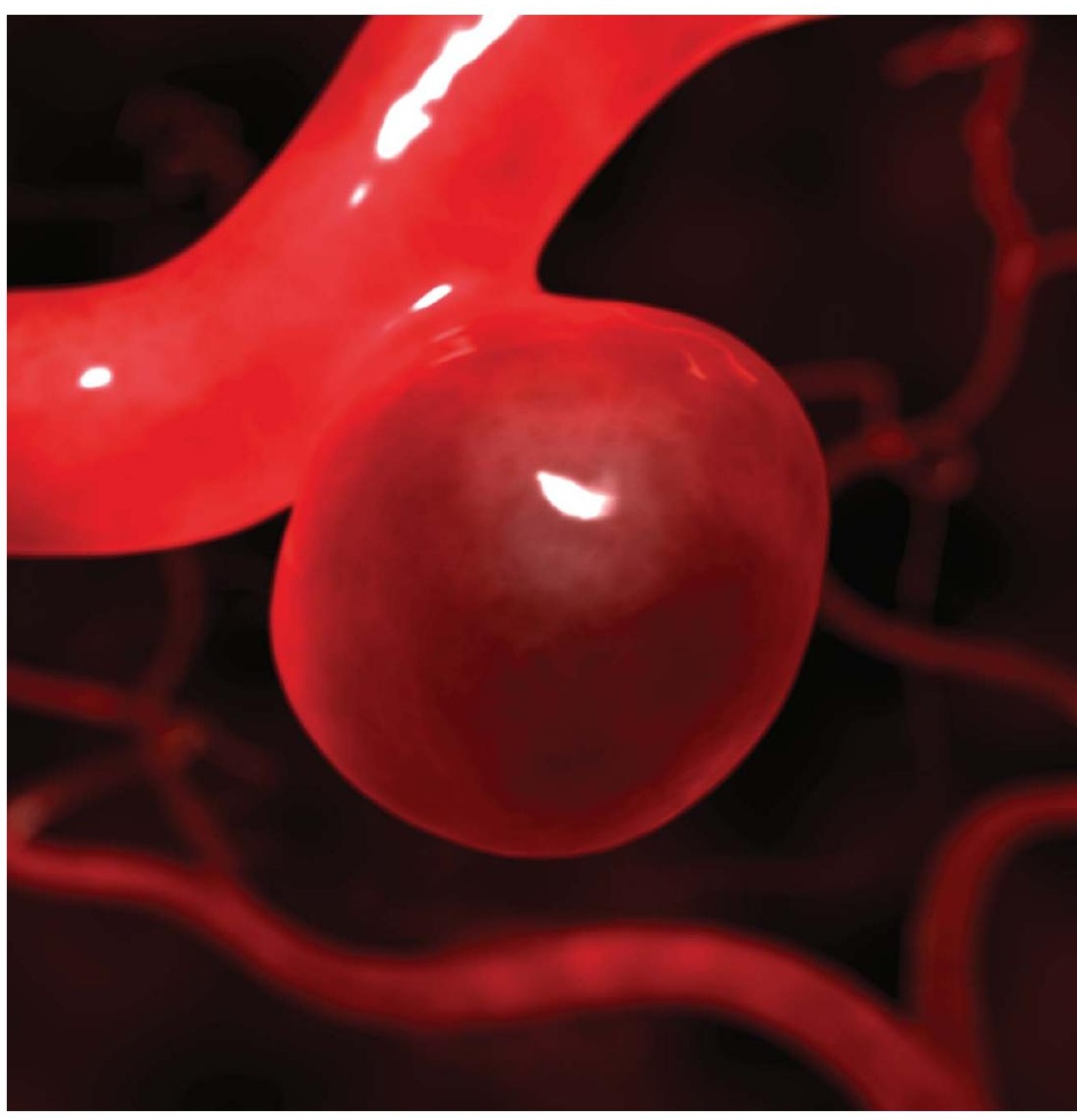
present in a variety of ways, from sudden death following subarachnoid hemorrhage to an incidental finding on cerebral imaging.

Aneurysm rupture with resultant subarachnoid hemorrhage (SAH) is the most feared consequence of unruptured intracranial aneurysms (UIA). SAH occurs at a rate of approximately 6-10/100,000 person-years, with ruptured aneurysms causing approximately $85 \% .^{2}$ The fatality rate for $\mathrm{SAH}$ is $30 \%-40 \%$, although this may be improving over time. ${ }^{3}$ Of those who survive $\mathrm{SAH}, 1$ in 5 may be functionally dependent. ${ }^{3}$ Even for those patients who obtain functional independence, a variety of psychological or neurologic sequelae can occur. ${ }^{2}$ Unruptured 
Figure 1 Common locations for intracranial saccular aneurysms

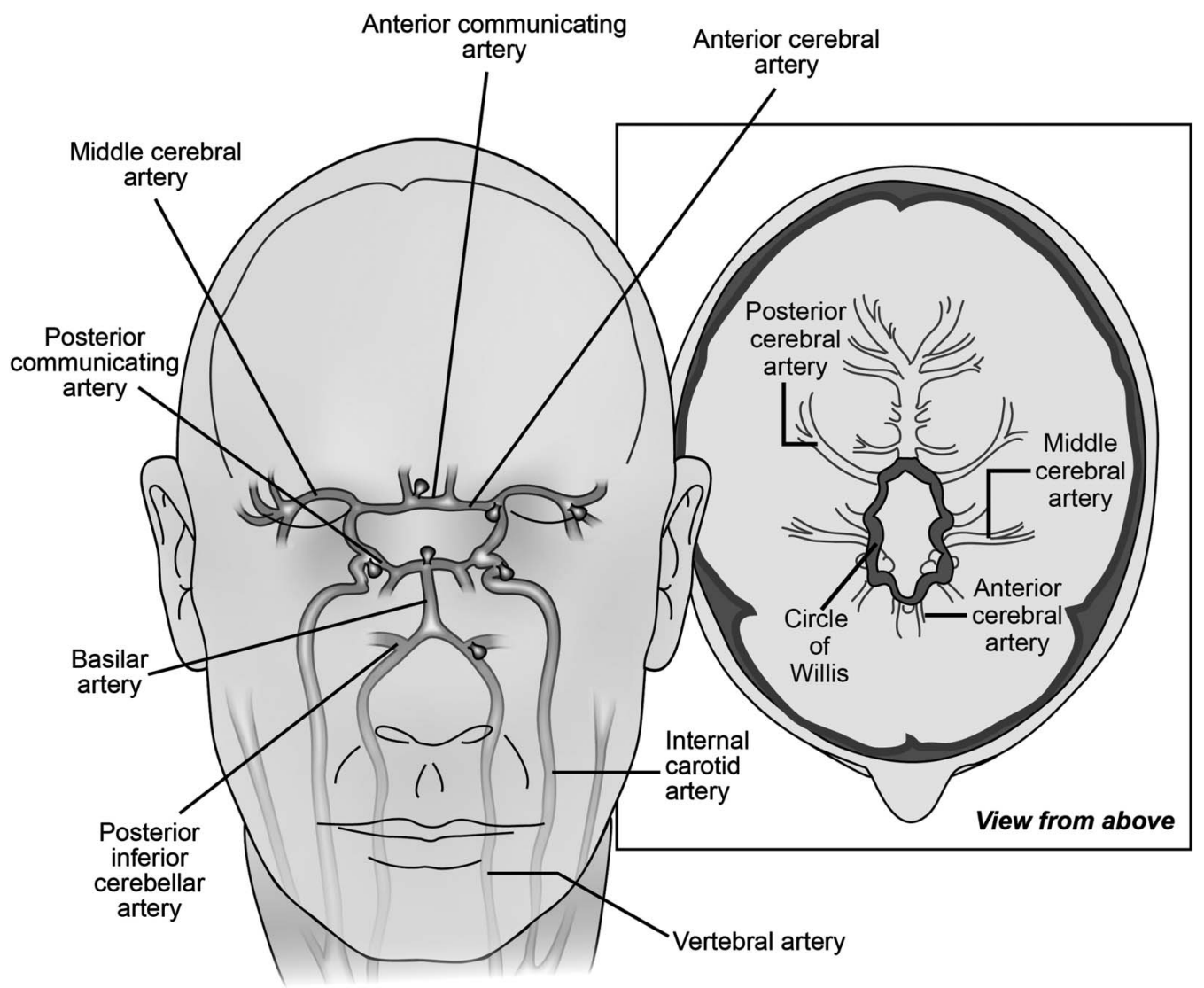

View from front

aneurysms can often remain asymptomatic (particularly those less than $10 \mathrm{~mm}$ ) or can present with symptoms including headache, seizure, cranial nerve palsies, focal neurologic deficits related to mass effect, and cerebral ischemic events distal to the site of aneurysm due to emboli arising from the aneurysmal sac. ${ }^{4}$

\section{Risk factors}

UIAs are common among the general population, occurring in $2 \%-3 \%$, but there are several factors that may increase one's risk. UIAs are seen more commonly in woman than men, particularly in those over 50 years of age, a group in which women are twice as likely to have UIAs. ${ }^{1}$ There appears to also be an increase in prevalence in older age groups. ${ }^{1}$ Certain medical conditions can increase the risk of aneurysms, including intracranial arteriovenous malformation, ${ }^{5}$ coarctation of the aorta, ${ }^{6}$ and fibromuscular dysplasia. ${ }^{7}$ Autosomal dominant polycystic kidney disease also carries an increased risk of UIA, with a recent study showing a prevalence of $12.4 \%$ ( $21.6 \%$ in those with family history of aneurysm or hemorrhagic stroke). ${ }^{8}$ Certain modifiable characteristics may place a person at increased risk for aneurysmal SAH including smoking, alcohol use, and hypertension. ${ }^{2}$

Family history of aneurysm and SAH is a particularly important risk factor for UIAs. Patients with one affected family member have approximately a $4 \%$ risk of having an UIA, which is slightly above the prevalence in the general population. ${ }^{9}$ If a patient has 2 or more affected first-degree family members, then the rate of UIAs increases $8 \%-10 \% .{ }^{10}$ Other variables such as female sex, hypertension, and smoking can further increase this risk. ${ }^{11}$ 
Table 1 Five-year cumulative rupture rate according to size and location of unruptured aneurysm from the International Study of Unruptured Aneurysms ${ }^{a}$

Aneurysm location

Cavernous carotid artery $(n=210), \%$

$\mathrm{AC} / \mathrm{MC} / \mathrm{IC}(\mathrm{n}=1,037), \%$

Post-P comm ( $=445)$, \%

\section{$<7 \mathrm{~mm}$ Group $1^{\text {b }}$}

0

0

2.5

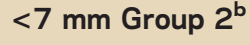

0

1.5

3.4

7-12 mm
0
2.6

14.5

13-24 mm

3.0

14.5

18.4 $\geq 25 \mathrm{~mm}$

6.4

40

50

Abbreviations: $\mathrm{AC}=$ anterior communicating or anterior cerebral artery; $I \mathrm{C}=$ internal carotid artery (not cavernous carotid artery); $\mathrm{MC}=$ middle cerebral artery; Post-P comm = vertebrobasilar, posterior cerebral arterial system, or the posterior communicating artery.

a Reprinted from Wiebers DO, Whisnant JP, Huston J 3rd, et al. Unruptured intracranial aneurysms: natural history, clinical outcome, and risks of surgical and endovascular treatment. Lancet 2003;362:103-110,12 with permission from Elsevier.

${ }^{b}$ Group 1 consists of those without prior subarachnoid hemorrhage; group 2 consists of those with prior subarachnoid hemorrhage from a separate aneurysm.

\section{Natural history}

In making treatment decisions, understanding the natural history of aneurysms is imperative. The International Study of Unruptured Intracranial Aneurysms (ISUIA) study group performed a large-scale prospective epidemiologic cohort study following 1,692 patients with UIAs that were $2 \mathrm{~mm}$ or larger $\left(1,077\right.$ without prior history of SAH). ${ }^{12}$ The overall annual rupture risk was $0.7 \%$. Size and location were important in predicting risk of rupture (table 1 ). Prior SAH slightly increased the risk of rupture for aneurysms less than $7 \mathrm{~mm} .{ }^{12}$ A prospective study from Japan reporting the natural history of patients with aneurysms $3 \mathrm{~mm}$ or larger showed an annual rupture rate of $0.95 \% .{ }^{13}$ As in prior studies, the risk of rupture increased with size, with a significant increase for aneurysms $7 \mathrm{~mm}$ or larger. Other risk factors for rupture included location on the anterior or posterior communicating artery and presence of a daughter sac. ${ }^{13}$ Another study followed 446 patients from Japan with aneurysms smaller than $5 \mathrm{~mm}$ for an average of 41 months. The rupture rate was $0.54 \%$ per year, with risk being greater for aneurysms greater than or equal to $4 \mathrm{~mm}$, patients younger than 50 years, those with hypertension, and those with multiple aneurysms. ${ }^{14}$ A large metaanalysis found that other factors including age over 60 years, female sex, Finnish or Japanese descent, aneurysm size over $5 \mathrm{~mm}$, posterior circulation location, and symptomatic UIA have a higher risk of rupture. ${ }^{15}$ Overall it appears that larger aneurysms have the greatest risk of rupture, but that other factors including aneurysm location and patient factors influence rupture risk as well.

\section{Screening}

A number of variables contribute to the delineation of appropriate screening parameters for a disease. Aneurysm rupture with SAH is a devastating disorder for which low-risk screening is available and which is potentially preventable with intervention. Based on this, one would consider that UIAs would be an optimal target for screening. However, most aneurysms are asymptomatic, often have a low rate of rupture, and interventions for aneurysms carry risk. In addition, there are important implications of screening for UIA, given that the detection of the aneurysm can cause significant anxiety and concern and may also lead to challenges in obtaining life or health insurance even though the risk of the aneurysm causing symptoms over the long term may be extremely low. While only limited information is available on the topic, widespread screening for low-risk groups is unlikely to provide any meaningful benefit. ${ }^{16-18}$ Consideration for screening should be restricted to those groups with higher risk of aneurysm and $\mathrm{SAH}$ and who would be candidates for intervention. Based on current information, it is reasonable to consider screening in firstdegree family members in families with 2 or more members with history of UIA or SAH; screening in those with only one affected family member is optional, and some first-degree 


\section{Consideration for screening should be} restricted to those groups with higher risk of aneurysm and SAH and who would be candidates for intervention.

relatives will request screening based on a relative's occurrence of SAH. ${ }^{16,17}$ It may also be reasonable to screen those with a family history of autosomal dominant polycystic kidney disease, particularly with family history of UIA or $\mathrm{SAH},{ }^{8}$ and in patients with coarctation of the aorta. ${ }^{6}$ If initial screening is negative for aneurysm, high-risk patients may need repeat screening in the future, with a suggested time interval of 5 years. ${ }^{19}$ Either brain magnetic resonance angiography (MRA) or CT angiography (CTA) may be used for screening, with both detecting aneurysms of $3 \mathrm{~mm}$ in diameter or greater with a high degree of sensitivity.

\section{Management}

When a UIA is detected, the available data regarding site- and size-specific natural history for the aneurysm detected should be carefully compared to the interventional risks. Three management options are available once an aneurysm is discovered: surgical clipping, endovascular treatment, or conservative management. While there are numerous published articles regarding UIA management, there is a lack of prospective randomized controlled trials to guide therapy, particularly in comparing intervention with observation. Many published articles are retrospective in nature, some are single-center, and many lack objective short- and long-term assessment of outcomes. ${ }^{20,21}$ Currently our best information regarding management of UIAs is based on observed rates of complications in aneurysm treatment compared to the natural history of UIAs or information extracted from the management of aneurysms in the setting of SAH.

\section{Surgical clipping}

Surgical clipping of aneurysms was introduced in 1937 by Dr. Walter Dandy, who used it to successfully treat a patient with a painful third nerve palsy caused by an internal carotid aneurysm. ${ }^{22}$ Today, the aneurysm is typically accessed through an open craniotomy, where the aneurysm is dissected out and a tiny metallic clip, which is selected based on the UIA anatomy, is placed at the neck and the aneurysm isolated from the parent blood vessel (figure 2).

Outcomes for aneurysmal clippings were assessed prospectively on 1,917 patients with UIA by the ISUIA study group. ${ }^{12}$ The 1 -year morbidity was $9.8 \%$ and mortality was $2.3 \%$. Risk of poor outcome for surgery was found to be increased with older age (risk increasing after age 50), aneurysm size greater than $12 \mathrm{~mm}$, aneurysm located in the posterior circulation, history of previous stroke, and aneurysm symptoms other than rupture.

Two large meta-analyses assessed UIA surgical clipping outcomes. One showed a morbidity rate of $4.1 \%$ and mortality rate of $1 \%$ and included a higher percentage of smaller and anterior circulation aneurysms. ${ }^{23}$ The other showed a morbidity rate of $10.9 \%$ with mortality rate of $2.6 \%$, but included a relatively high number of posterior circulation aneurysms $(29.7 \%)$ and giant aneurysms (27.1\%). ${ }^{24}$ These meta-analyses represent the cumulation of published numbers on morbidity and mortality, which may not be representative of the true morbidity and mortality occurring from these surgeries in most clinical settings. Surgical clipping appears to generally be effective with complete occlusion obtained in greater than $90 \%$ of cases. ${ }^{25}$

\section{Endovascular coiling}

Endovascular management emerged as a treatment modality in the 1990s and has been increasing in popularity since that time. ${ }^{26,27}$ This procedure is now more common than surgery for 
Figure 2 Aneurysm interventions

A
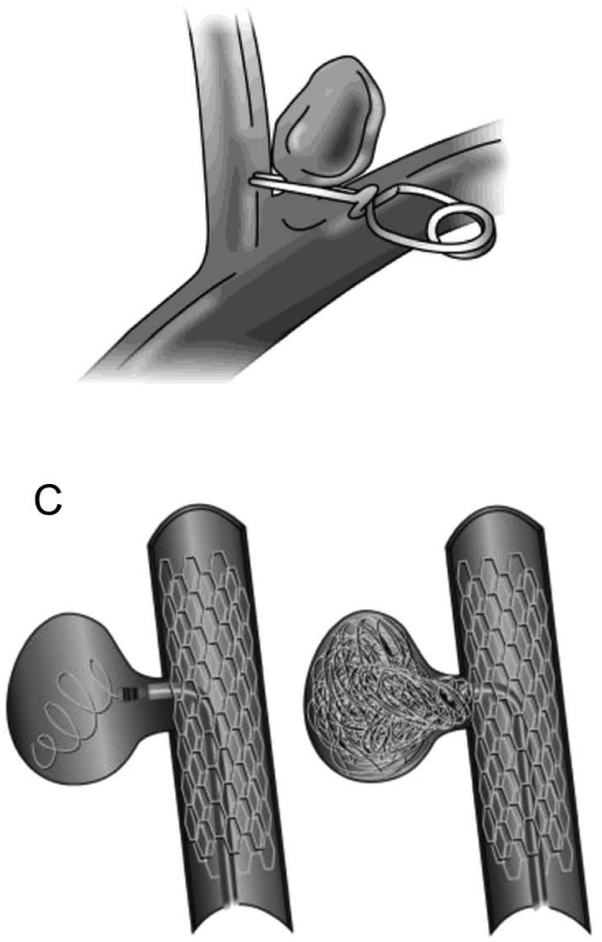

B

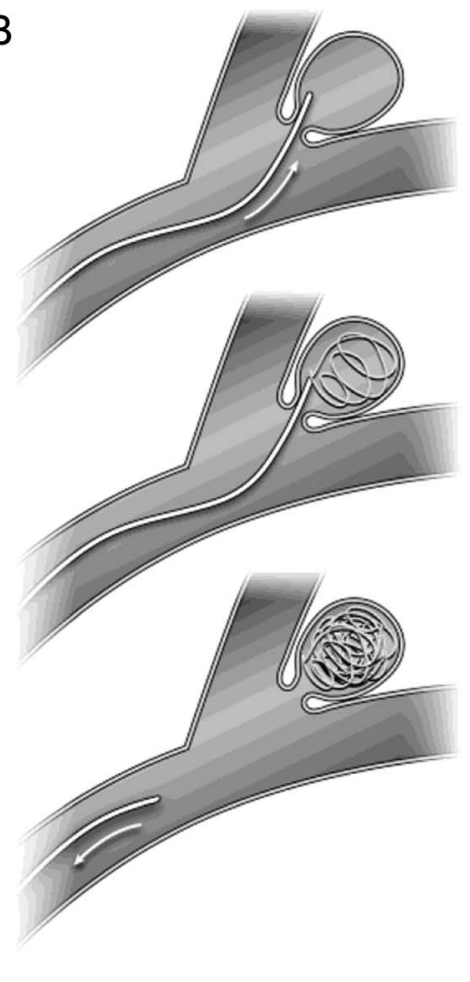

(A) Aneurysm clipping. (B) Endovascular coiling. (C) Endovascular coiling with stent assistance.

management of UIA in the United States. ${ }^{26}$ In the most common form of endovascular management, platinum coils are introduced into the aneurysm, causing local thrombosis and isolation of the aneurysm from the parent artery. Certain cases may not be candidates for this form of intervention including large aneurysms, aneurysms with a wide neck, high dome-to-neck ratio, or those with difficult intravascular approaches. ${ }^{27}$ Adjunct techniques such as balloon inflation or stent placement at the aneurysm neck are increasingly used in some of these more difficult cases (figure 2) ${ }^{20}$ Other endovascular techniques include use of flow diverting stents and use of liquid embolic agents, which are typically used for large aneurysms. ${ }^{20}$

For endovascular management, the risk of unfavorable outcomes is approximately $4 \%-5 \%$, with a risk of mortality of $1 \%-2 \%$ based on review and meta-analysis of existing literature. $^{20,21}$ The rate of poor outcomes with endovascular procedures appears to be decreasing over time, but the characteristics of aneurysms treated by endovascular procedures are also changing over time, making it difficult to determine if this change is due to improvement of technique or a change in patient population. ${ }^{20}$ Aneurysms are successfully occluded $86.1 \%$ of the time based on postprocedure imaging, with recurrence in $24.4 \%$ and need for retreatment in $9.1 \%{ }^{21}$ The ISUIA study group also assessed outcomes prospectively for endovascular coiling in 451 patients. The 1-year morbidity rate was $6.4 \%$ and the mortality rate was $3.1 \% .{ }^{12}$ Of note, as the baseline characteristics of this group were different from the surgery group (including older patients, larger aneurysms, and larger number of posterior circulation aneurysms), the results are not directly comparable. The risk of poor outcome with endovascular procedure was higher with aneurysm diameter greater than $12 \mathrm{~mm}$ and posterior circulation location.

As there are no randomized trials to directly compare management options for UIAs, one can consider clinical trials regarding the management of ruptured aneurysms for further 


\section{Three management options are available once an aneurysm is discovered: surgical clipping, endovascular treatment, or conservative} management.

information. The International Subarachnoid Aneurysm Trial directly compared surgical clipping to endovascular coiling for ruptured intracranial aneurysms in a prospective randomized trial. ${ }^{28}$ In this study, the risk of death or dependency was lower in the endovascular treatment group (23.5\%) compared to the neurosurgical group (30.9\%), with a risk reduction of $7.4 \%$ that was statistically significant. ${ }^{28}$

\section{Who should be treated?}

The decision regarding whether to manage a UIA with an interventional procedure is complex. Available natural history data suggest that many patients are asymptomatic and may remain asymptomatic for a long period of time, if not indefinitely. Intervention has a high rate of success, but carries risk (albeit low) of immediate disability or death. Existing guidelines were released before of many of the commonly cited prospective studies on natural history and outcomes for contemporary interventional treatment were published. ${ }^{16}$ Furthermore, there are no randomized controlled trials to guide management.

Based on the risks and benefits, most symptomatic aneurysms or those larger than $12 \mathrm{~mm}$ should undergo interventional management unless a patient's medical condition would place them at high risk for morbidity and mortality from a procedure or their lifespan was limited. Cavernous internal carotid aneurysms do not require intervention unless they become symptomatic, as they are unlikely to result in SAH due to their extradural location. Aneurysms less than $7 \mathrm{~mm}$ are reasonable to observe with repeat imaging unless the patient is young, particularly with other risk factors such as prior $\mathrm{SAH}$, family history of $\mathrm{SAH}$, location in posterior circulation or posterior communicating artery, or presence of a daughter sac. Optimal management of aneurysms between 7 and $12 \mathrm{~mm}$ is unclear and should be considered on a case-by-case basis based on patient and aneurysm risk factors, intervention options and risks, and patient age. For all patients, their perspective regarding treatment after hearing a summary of the natural history and interventional risks is of utmost importance. Based on the available data, for those aneurysms for which an interventional procedure is recommended, endovascular management may be preferable, with lower rate of complications and mortality, although both methods appear efficacious. If an aneurysm is recommended for conservative management, then our practice is to repeat noninvasive imaging at 6 months after diagnosis, and then annually for 3 years, with subsequent decrease in intervals if stable. There is a risk of aneurysm growth during follow-up, with this risk dependent on the initial UIA diameter. One study using MRA follow-up of UIA for an average of 4 years reported a risk of growth of $6.9 \%$ for a UIA less than $8 \mathrm{~mm}, 25 \%$ for $8-12 \mathrm{~mm}$, and $83 \%$ for greater than $12 \mathrm{~mm}$ in diameter. ${ }^{29}$ In general, UIA growth leads to a recommendation for interventional treatment given the possible increased risk of SAH if the aneurysm is not of stable size.

Risk factor reduction should also be recommended for all patients with a UIA, including optimal blood pressure control, avoidance of cigarette smoking, and moderation of alcohol use.

\section{Antiplatelet and anticoagulant use}

Antiplatelet agents and anticoagulants are commonly used medications that may present a dilemma in UIA management. Few data are available regarding their safety in patients with a UIA. A population-based case-control study in Denmark showed an increased association between SAH and dipyridamole use and new aspirin use, but not long-term aspirin use. ${ }^{30}$ 
Table 2 Summary of recommendations

\section{Recommendations for screening}

Strongly consider screening

- Patients with 2 or more family members with history of UIA or $\mathrm{SAH}$

- ADPKD with family history of UIA or SAH

- Patients with coarctation of the aorta

\section{Recommendations for treatment}

Strongly consider treatment with clipping or endovascular procedure

- UIA $\geq 12 \mathrm{~mm}$ in diameter

- Symptomatic UIA

- Enlarging UIA

\section{Possibly consider screening}

- Patients with ADPKD without family history of aneurysm

- Patients with one family member with UIA or SAH (per patient preference)

\section{Do not recommend screening}

- General population
- UIA in younger patients

\section{Possibly consider treatment with clipping or endovascular procedure}

- $7 \mathrm{~mm} \leq \mathrm{UIA}<12 \mathrm{~mm}$ in diameter + any of the following features:

- UIA in higher-risk locations such as posterior circulation or posterior communicating artery

- UIA with daughter sac

Family history of SAH

- UIA $<7 \mathrm{~mm}$ in diameter in younger patients + any of the following features:

UIA in high-risk locations such as posterior circulation or posterior communicating artery

- UIA with daughter sac

Family history of SAH

Abbreviations: ADPKD = autosomal dominant polycystic kidney disease; $\mathrm{SAH}=$ subarachnoid hemorrhage; UIA $=$ unruptured intracranial aneurysm.

A case-control study utilizing the ISUIA cohort suggested that aspirin use may have a protective affect against rupture. ${ }^{31}$ Aspirin use prior to rupture does not appear to worsen outcomes from SAH. ${ }^{32}$ We generally recommend that antiplatelet agents be used as necessary in patients with a UIA based on the patient's specific indication for such medication. In regard to anticoagulants, their use has been associated with a poorer outcome from $\mathrm{SAH},{ }^{33}$ but not clearly associated with an increased risk for aneurysm rupture. ${ }^{12,34}$ Anticoagulation may be necessary in some patients with UIA, but there should be careful consideration of risks and benefits and a thorough discussion with the patient. Definitive management of the aneurysm through clipping or coiling may need to be considered in some situations.

\section{Accessibility concerns}

The aforementioned recommendations regarding screening, monitoring, and management of UIA are critically dependent on easy accessibility to radiologic and surgical resources. This may not always be the case for all patients or all medical systems, particularly those in developing countries. Difficulty in monitoring may push one more toward definitive intervention for UIA. However, if surgical/procedural resources are limited, this can potentially have a 
negative impact on morbidity and mortality for intervention given that data suggest that higher-volume centers likely have the lowest risk of an adverse outcome. A change in the risk profile for the intervention would consequently alter recommendations regarding screening and intervention.

\section{DISCUSSION}

UIAs are common in the general population and, with the increase in detection on noninvasive imaging, are likely to be encountered by all clinicians. Many UIAs may have a benign course, but carry a risk for rupture, with potentially catastrophic SAH. Currently, there are 2 effective methods for treatment, including surgical clipping and endovascular management, but such treatments carry a risk of morbidity and mortality. When an unruptured aneurysm is detected, the aneurysm's natural history is compared to interventional risks, with a consideration of numerous aneurysm and patient factors. These include aneurysm size, location, morphology, and presentation and patient factors including age, family history of $\mathrm{SAH}$, personal history of $\mathrm{SAH}$, presence of medical comorbidities, and their perspective regarding an interventional procedure. Widespread screening is generally not recommended, unless there is a history of 2 or more family members with aneurysms or SAH, or in select medical conditions. A summary of screening and management recommendations is found in table 2.

\section{REFERENCES}

1. Vlak MH, Algra A, Brandenburg R, Rinkel GJ. Prevalence of unruptured intracranial aneurysms, with emphasis on sex, age, comorbidity, country, and time period: a systematic review and meta-analysis. Lancet Neurol 2011;10:626-636.

2. van Gijn J, Kerr RS, Rinkel GJ. Subarachnoid haemorrhage. Lancet 2007;369:306-318.

3. Nieuwkamp DJ, Setz LE, Algra A, Linn FH, de Rooij NK, Rinkel GJ. Changes in case fatality of aneurysmal subarachnoid haemorrhage over time, according to age, sex, and region: a meta-analysis. Lancet Neurol 2009;8:635-642.

4. Raps EC, Rogers JD, Galetta SL, et al. The clinical spectrum of unruptured intracranial aneurysms. Arch Neurol 1993;50:265-268.

5. Brown RD Jr, Wiebers DO, Forbes GS. Unruptured intracranial aneurysms and arteriovenous malformations: frequency of intracranial hemorrhage and relationship of lesions. J Neurosurg 1990;73:859-863.

6. Connolly HM, Huston J 3rd, Brown RD Jr, Warnes CA, Ammash NM, Tajik AJ. Intracranial aneurysms in patients with coarctation of the aorta: a prospective magnetic resonance angiographic study of 100 patients. Mayo Clin Proc 2003;78:1491-1499.

7. Olin JW, Sealove BA. Diagnosis, management, and future developments of fibromuscular dysplasia. J Vasc Surg 2011;53:826-836.

8. Xu HW, Yu SQ, Mei CL, Li MH. Screening for intracranial aneurysm in 355 patients with autosomal-dominant polycystic kidney disease. Stroke 2011;42:204-206.

9. Raaymakers TW. Aneurysms in relatives of patients with subarachnoid hemorrhage: frequency and risk factors: MARS Study Group: magnetic resonance angiography in relatives of patients with subarachnoid hemorrhage. Neurology 1999;53:982-988.

10. Ronkainen A, Miettinen H, Karkola K, et al. Risk of harboring an unruptured intracranial aneurysm. Stroke 1998;29:359-362.

11. Brown RD Jr, Huston J, Hornung R, et al. Screening for brain aneurysm in the Familial Intracranial Aneurysm study: frequency and predictors of lesion detection. J Neurosurg 2008;108:1132-1138.

12. Wiebers DO, Whisnant JP, Huston J 3rd, et al. Unruptured intracranial aneurysms: natural history, clinical outcome, and risks of surgical and endovascular treatment. Lancet 2003;362:103-110.

13. Morita A, Kirino T, Hashi K, et al. The natural course of unruptured cerebral aneurysms in a Japanese cohort. N Engl J Med 2012;366:2474-2482.

14. Sonobe M, Yamazaki T, Yonekura M, Kikuchi H. Small unruptured intracranial aneurysm verification study: SUAVe study, Japan. Stroke 2010;41:1969-1977.

15. Wermer MJ, van der Schaaf IC, Algra A, Rinkel GJ. Risk of rupture of unruptured intracranial aneurysms in relation to patient and aneurysm characteristics: an updated meta-analysis. Stroke 2007;38:1404-1410.

16. Bederson JB, Awad IA, Wiebers DO, et al. Recommendations for the management of patients with unruptured intracranial aneurysms: a statement for healthcare professionals from the Stroke Council of the American Heart Association. Circulation 2000;102:2300-2308. 
17. The Magnetic Resonance Angiography in Relatives of Patients with Subarachnoid Hemorrhage Study Group. Risks and benefits of screening for intracranial aneurysms in first-degree relatives of patients with sporadic subarachnoid hemorrhage. N Engl J Med 1999;341:1344-1350.

18. Wermer MJ, Buskens E, van der Schaaf IC, Bossuyt PM, Rinkel GJ. Yield of screening for new aneurysms after treatment for subarachnoid hemorrhage. Neurology 2004;62:369-375.

19. Rinkel GJ. Intracranial aneurysm screening: indications and advice for practice. Lancet Neurol 2005; 4:122-128.

20. Naggara ON, Lecler A, Oppenheim C, Meder JF, Raymond J. Endovascular treatment of intracranial unruptured aneurysms: a systematic review of the literature on safety with emphasis on subgroup analyses. Radiology 2012;263:828-835.

21. Naggara ON, White PM, Guilbert F, Roy D, Weill A, Raymond J. Endovascular treatment of intracranial unruptured aneurysms: systematic review and meta-analysis of the literature on safety and efficacy. Radiology 2010;256:887-897.

22. Louw DF, Asfora WT, Sutherland GR. A brief history of aneurysm clips. Neurosurg Focus 2001; 11:E4.

23. King JT Jr, Berlin JA, Flamm ES. Morbidity and mortality from elective surgery for asymptomatic, unruptured, intracranial aneurysms: a meta-analysis. J Neurosurg 1994;81:837-842.

24. Raaymakers TW, Rinkel GJ, Limburg M, Algra A. Mortality and morbidity of surgery for unruptured intracranial aneurysms: a meta-analysis. Stroke 1998;29:1531-1538.

25. David CA, Vishteh AG, Spetzler RF, Lemole M, Lawton MT, Partovi S. Late angiographic follow-up review of surgically treated aneurysms. J Neurosurg 1999;91:396-401.

26. Brinjikji W, Rabinstein AA, Nasr DM, Lanzino G, Kallmes DF, Cloft HJ. Better outcomes with treatment by coiling relative to clipping of unruptured intracranial aneurysms in the United States, 2001-2008. AJNR Am J Neuroradiol 2011;32:1071-1075.

27. Dovey Z, Misra M, Thornton J, Charbel FT, Debrun GM, Ausman JI. Guglielmi detachable coiling for intracranial aneurysms: the story so far. Arch Neurol 2001;58:559-564.

28. Molyneux AJ, Kerr RS, Yu LM, et al. International subarachnoid aneurysm trial (ISAT) of neurosurgical clipping versus endovascular coiling in 2143 patients with ruptured intracranial aneurysms: a randomised comparison of effects on survival, dependency, seizures, rebleeding, subgroups, and aneurysm occlusion. Lancet 2005;366:809-817.

29. Burns JD, Huston J 3rd, Layton KF, Piepgras DG, Brown RD Jr. Intracranial aneurysm enlargement on serial magnetic resonance angiography: frequency and risk factors. Stroke 2009;40:406-411.

30. Schmidt M, Johansen MB, Lash TL, Christiansen CF, Christensen S, Sorensen HT. Antiplatelet drugs and risk of subarachnoid hemorrhage: a population-based case-control study. J Thromb Haemost 2010;8:1468-1474.

31. Hasan DM, Mahaney KB, Brown RD Jr, et al. Aspirin as a promising agent for decreasing incidence of cerebral aneurysm rupture. Stroke 2011;42:3156-3162.

32. Toussaint LG 3rd, Friedman JA, Wijdicks EF, et al. Influence of aspirin on outcome following aneurysmal subarachnoid hemorrhage. J Neurosurg 2004;101:921-925.

33. Rinkel GJ, Prins NE, Algra A. Outcome of aneurysmal subarachnoid hemorrhage in patients on anticoagulant treatment. Stroke 1997;28:6-9.

34. Tarlov N, Norbash AM, Nguyen TN. The safety of anticoagulation in patients with intracranial aneurysms. J Neurointerv Surg Epub 2012 Jun 7.

\section{STUDY FUNDING}

No targeted funding reported.

\section{DISCLOSURES}

The authors report no disclosures relevant to the manuscript. Go to Neurology.org/cp for full disclosures. 


\section{Related articles from other AAN physician and patient resources}

Neurology ${ }^{\circledR} \quad \bullet \quad$ www.neurology.org

Radiosurgery for unruptured cerebral arteriovenous malformations: Long-term seizure outcome March 28, 2012;78:1292-1298.

Outcomes and prognostic factors of intracranial unruptured vertebrobasilar artery dissection May 17, 2011;76:1735-1741.

\section{Neurology Now ${ }^{\circledR}$ • www.neurologynow.com}

Hidden Hercules: Actor Kevin Sorbo reveals his secret battle with stroke October/November 2011;26-31.

\section{Neurology Today ${ }^{\circledR} \quad$ • www.neurotodayonline.com}

FDA approves new system for brain aneurysm repair June 16, 2011;11:24-25.

Intracranial stenting's risks over medical therapy prompt halt of major trial May 5, 2011:11:10.

Interventional options remain risky for brain arteriovenous malformations December 1, 2011;11:18-21. 